\title{
Publisher's Note: “All-optical delay line using semiconductor cavity solitons" [Appl. Phys. Lett. 92, 011101 (2008)]
}

\author{
F. Pedaci, ${ }^{1}$ S. Barland,${ }^{1}$ E. Caboche,${ }^{1}$ P. Genevet, ${ }^{1}$ M. Giudici, ${ }^{1}$ J. R. Tredicce,,${ }^{1, a)}$ \\ T. Ackemann, ${ }^{2}$ A. J. Scroggie, ${ }^{2}$ W. J. Firth, ${ }^{2}$ G.-L. Oppo, ${ }^{2}$ G. Tissoni, ${ }^{3}$ and R. Jäger ${ }^{4}$ \\ ${ }^{1}$ Institut Non-linéaire de Nice, Université de Nice Sophia, Antipolis-Centre Nationale de la Recherche \\ Scientifique, 1361 route des Lucioles, 06560 Valbonne, France \\ ${ }^{2}$ SUPA, Department of Physics, University of Strathclyde, 107 Rottenrow, Glasgow G4 ONG, Scotland, \\ United Kingdom \\ ${ }^{3}$ INFM-CNR and CNISM, Dipartimento di Matematica e Fisica, Università dell'Insubria, Via Valleggio 11, \\ 22100 Como, Italy \\ ${ }^{4}$ ULM Photonics, Lise Meitner Str. 13, 89081 Ulm, Germany
}

(Received 5 June 2009; published online 29 June 2009)

[DOI: $10.1063 / 1.3168395]$

This article was originally published online and in print with an error in the deposit number for AIP's Electronic Physics Auxiliary Publication Service (EPAPS): In Ref. 17, the EPAPS Deposit No. E-APPLAB-92-01801 should have been E-APPLAB-92-010801. AIP apologizes for this error. All online versions of the article have been corrected.

${ }^{a)}$ Electronic mail: jorge.tredicce@inln.cnrs.fr. 